\title{
Fractional CO2 Laser for the Treatment of Vulvar Lichen Sclerosus: a Double-Blind, Sham-Controlled Randomized Trial
}

\author{
Leia Mitchell ${ }^{1}$, Andrew Goldstein ${ }^{2}$, Debra Heller ${ }^{3}$, Theodora Mautz ${ }^{2}$, Chelsea Thorne ${ }^{2}$, So \\ Yeon Joyce Kong ${ }^{1}$, Maria Sophocles ${ }^{4}$, Hillary Tolson ${ }^{2}$, and Jill Krapf ${ }^{2}$ \\ ${ }^{1}$ Mercer University College of Continuing and Professional Studies \\ ${ }^{2}$ The Center for Vulvovaginal Disorders \\ ${ }^{3}$ Rutgers New Jersey Medical School \\ ${ }^{4}$ Women's Healthcare of Princeton
}

October 5, 2020

\begin{abstract}
Objective To determine the efficacy of fractional carbon dioxide laser (FXCO2) therapy for vulvar lichen sclerosus (VLS). Design Prospective, double-blind, sham-controlled, randomized trial. Setting Clinic specializing in vulvovaginal disorders. Population Forty women with active VLS confirmed with biopsy who abstaining from topical and/or systemic treatments for at least 4 weeks. Methods Women were randomized in a 1:1 ratio to receive either five sham laser treatments or five FXCO2 treatments in a 24-week period. Pre- and post-treatment biopsies were obtained on all participants. Study participants, treating clinicians, and evaluating pathologist were blinded. Main Outcome Measures Primary: pre- and post-treatment biopsy Histopathologic Scale (HS) findings. Secondary: Clinical Scoring System for Vulvar Lichen Sclerosus (CSS) Results There was a 0.12 reduction (improvement) in HS from baseline in the active treatment group $(95 \% \mathrm{CI}=-1.01,0.78, \mathrm{p}=0.79)$ and a 0.06 increase from baseline in the sham treatment group $(95 \% \mathrm{CI}--0.81,0.92, \mathrm{p}=0.90)$. The change in HS between the active and sham arm was not statistically significant $(-0.17 ; 95 \% \mathrm{CI}=-1.14,1.06, \mathrm{p}=0.78)$. There was a 6.82 -point reduction (improvement) in the patients' CSS from baseline in the active $(95 \% \mathrm{CI}=-11.28,-2.37, \mathrm{p}=0.004)$ and a 4.83 -point reduction in the sham treatment group (95\% CI $=-9.16,-0.51, \mathrm{p}=0.03$ ). In the clinicians' CSS, there was a 0.82 increase (worsening) in the active $(95 \% \mathrm{CI}=$ $-0.46,2.11, \mathrm{p}=0.20)$ and a 0.28 reduction in the sham treatment group $(95 \% \mathrm{CI}=-1.53,0.97, \mathrm{p}=0.65)$. Conclusions. FXCO2 is not an effective monotherapy treatment for VLS
\end{abstract}

\section{Tweetable abstract:}

Fractional $\mathrm{CO}_{2}$ laser treatments are not more effective than sham treatments for vulvar lichen sclerosus

\section{Keywords:}

Autoimmune disorders

Dermatology

Dyspareunia

Gynecology

Lichenification

Lichenoid

Microablative therapy 
Sexual dysfunction

Vulvar carcinoma

Vulvar dermatoses

Vulvar lichen sclerosus

\section{Introduction}

Vulvar lichen sclerosus (VLS) is a chronic cutaneous disorder affecting approximately one in 70 women. ${ }^{1}$ Presenting symptoms may include intense pruritus, pain, burning, and severe dyspareunia. The typical lesions of VLS are white plaques and papules, often with areas of ecchymosis, excoriation, and ulceration, with destruction of the vulvar architecture. Four to seven percent of women with VLS develop vulvar carcinoma. ${ }^{2}$ The histopathologic changes of VLS are distinctive, making biopsy a very useful diagnostic tool. ${ }^{3}$ While there is no known cure for VLS, the current gold standard treatment is topical ultrapotent corticosteroids. ${ }^{4,5}$ When properly administered, topical ultrapotent corticosteroids help to resolve symptoms and prevent further vulvar scarring. Proper treatment with corticosteroids can reverse underlying histopathologic inflammation and decrease risk of malignant transformation. ${ }^{6}$ Although treatment with topical corticosteroids is effective, these medications have the potential for serious local and systemic side effects, including dermal thinning, skin atrophy, superimposed infections, rebound dermatitis, and adrenal insufficiency. ${ }^{7}$ Even though the aforementioned side effects are uncommon, these concerns have prompted interest in alternative interventions for VLS.

Recently, microablative fractional $\mathrm{CO}_{2}$ laser $\left(\mathrm{FxCO}_{2}\right)$ has been proposed for the management of VLS. This type of laser has a wavelength of $10,600 \mathrm{~nm}$ allowing a superficial microablative effect in soft tissues, as well as a pulsed beam, which protects tissues from possible damage due to overheating. The laser beam is delivered to the tissue in a fractional manner, creating small spots $(150-200 \mu \mathrm{m})$ alternating parts of tissue treated and not treated (Figure 2). The microablation stimulates remodeling of the connective tissue via the production of heat shock protein 47 and produces new collagen/fibroblasts and ground matrix.

Multiple small non-controlled studies and case series have indicated $\mathrm{FxCO}_{2}$ therapy as a promising treatment modality to treat VLS. ${ }^{-1}$ These studies suggest that $\mathrm{FxCO}_{2}$ treatment may stimulate protein synthesis, accelerate tissue reconstruction, and decrease lichenification. Furthermore, the clinical manifestations of LS, such as intense vulvar pruritus and burning, appear to improve. However, none of these studies were blinded or placebo-controlled. In many of the studies, topical corticosteroids were used either concurrently or immediately prior to laser treatment. In addition, a number of these studies did not measure histologic changes prior to and after laser treatment and relied instead on subjective reporting and clinical observation. The current study evaluates the efficacy of $\mathrm{FXCO}_{2}$ as a monotherapy treatment for VLS in a randomized, blinded, sham-controlled trial.

\section{Methods}

IRB approval was obtained from Advarra IRB (Pro00024516) on March 1, 2018 and the study was listed on www.clinicaltrials.gov (NCT03665584). There was no formal patient and public involvement (PPI) in this research. Partial funding for this study was obtained from the Gynecologic Cancer Research Foundation, a non-profit Maryland, USA corporation. El.En Group, Florence, Italy, the manufacturer of the laser used in this study provided additional funding and supplied the laser used in the study. El.En Group had no role in the analysis and interpretation of data, in the writing of the report, or in the decision to submit the article for publication.

Women with a diagnosis of biopsy-proven active VLS were recruited from one center that specializes in the treatment of vulvovaginal disorders. The study timeline consisted of a four-week (minimum) washout period, a two-week screening period, a 24 -week treatment period, and an 8 week post-treatment period. For the washout period, participants were required to stop all topical corticosteroids, topical immunosuppressants, and any other medications used to treat VLS. In the screening period, a 4-millimeter punch skin biopsy was 
collected from each patient to confirm the diagnosis of active lichen sclerosus and to rule out the diagnoses of lichen planus, psoriasis, and vulvar intraepithelial neoplasia (VIN). A vulvoscopy was performed at the screening visit and after the 24-week treatment period to rule out vulvar carcinoma or VIN. All eligible patients were randomized to receive either 5 sham treatments $\left(\mathrm{FxCO}_{2}\right.$ with very minimal laser energy emitted) or 5 active treatments of $\mathrm{FxCO}_{2}$ (with laser energy emitted). A repeat biopsy was performed adjacent to the original biopsy site 8 weeks after the final treatment.

Randomization was performed by a research assistant using a random number generator (available at www.sealedenvelop.com). Randomization was a 1:1 ratio of active to sham treatment.

The $\mathrm{FxCO}_{2}$ treatment was performed at baseline and then repeated at 4 week intervals for a total of 5 treatments, using a protocol recommended by the laser's manufacturer (Supplement Table 3). The sham treatment was intentionally designed to deliver a very small amount of laser energy to this tissue. There is a patient awareness shared on VLS social media groups that $\mathrm{FXCO}_{2}$ produces visible spots on the skin and creates smoke and odor from skin vaporization. The amount of energy used in the sham group was enough to create the spots, smoke, and odor so that both the patients and investigator remained blinded, but not strong enough to affect the pathological process.

The primary efficacy endpoint was improvement in the histopathologic changes of VLS in biopsy specimens obtained during the screening period and after the 24 -week treatment period. The histopathologic changes were evaluated by a blinded expert gynecologic pathologist (DH), who quantified the severity of the changes on a 0 to 6 point histopathology scale (HS). This HS quantifies the loss of rete pegs, the amount of dermal homogenization, and the amount of chronic inflammation. A secondary endpoint was the change from baseline in the Clinical Scoring System for Vulvar Lichen Sclerosus (CSS) a validated instrument that assesses both an investigator's impression of the severity of disease and a patient's impression of the severity of her symptoms. ${ }^{1}$ The patients' subjective section of the CSS is scored from 0-40 and is the total of the 4 domains: pruritus, soreness, burning, and dyspareunia. The clinicians' objective section of the CSS is scored from 0-12 and is the sum of 6 domains: fissures, erosions, hyperkeratosis, agglutination, stenosis, and atrophy.

\section{Statistical Analysis}

A sample size calculation was performed prior to the onset of the study and the sample size of 36 subjects was determined to show a clinically significant $40 \%$ reduction in the histopathology scale with a two-sided significance of 0.05 and a power of 0.8 .

Differences in baseline characteristics by treatment group were analyzed with Wilcoxon signed-rank test for continuous variables (age and years since symptom) and Fisher's exact tests for categorical variable (race). The mean clinical outcomes were adjusted for age, race, and years since symptomatic. Longitudinal CCS were modeled with repeated-measures linear regression models as implemented in the SAS procedure PROC MIXED (version 9.45).

The analysis of study outcomes was based on linear mixed models (PROC MIXED) for patient CSS, provider CSS, and HS score accounting for the covariates (age, race, and years since symptom), and an unstructured covariance matrix was assumed. Changes over time in the CCS and HS scores (differences between third session scores and baseline scores) between treatment and control groups were also assessed (difference-indifference method).

Results of the PROC MIXED models are reported as adjusted means (calculated from least-square means) and the $95 \%$ confidence intervals (CIs). All statistical analysis was performed using SAS software (version 9.4; SAS Institute, Inc, Cary, NC).

\section{Results}

This study was conducted between November, 2018 and June, 2020. 305 women with VLS from a patient database were screened for eligibility. As shown in Figure, 50 women met initial inclusion criteria and agreed to participate in the study. Ten women did not have active disease on examination or pretreatment biopsy 
and were, therefore, excluded prior to treatment. Forty women were randomized: 20 were assigned to receive $\mathrm{FxCO}_{2}$ laser and 20 were assigned the sham laser. Thirty-five women were included in the study analysis, 17 of which received treatment and 18 received sham. Of the five participants not included in the final analysis, three women had missed 2 or more treatments due to COVID-19 travel restrictions and/or office closures and were, therefore, not included in the study analysis, one woman was removed from the study due to a severe exacerbation in the symptoms and signs of VLS after receiving only two laser treatments, and one woman voluntarily withdrew due to an exacerbation of the severity of her symptoms. Eighty-six percent (31) of the participants included in study analysis were Caucasian, 2.8\% (1) was African American, and 8.6\% (3) were Latina, Brazilian, or Spanish. The median age of the women at first visit was 59 years old and there were no significant differences between study groups in regard to age, race, or duration of symptoms (Table 1).

The histopathological scale (HS) is a 0-6 scale. The model-based mean HS prior to treatment in the sham group was 4.07 and the model-based mean HS score in the treatment group was 4.26. This difference was not statistically significant.

As shown in Table 2, there was a 0.12 reduction (improvement) in the $\mathrm{HS}$ from baseline in the $\mathrm{FXCO}_{2}$ treatment group $(95 \% \mathrm{CI}=-1.01,0.78, \mathrm{p}$-value $=0.79)$ and a 0.06 increase in the HS from baseline in the sham treatment group $(95 \% \mathrm{CI}=-0.81,0.92, \mathrm{p}$-value $=0.90)$. The change in HS score between the active treatment arm and the sham arm was $-0.17(95 \% \mathrm{CI}=-1.14,1.06)$. This difference was not statistically significant with a p-value of 0.78 .

Based on the model-based mean, there was a $6.82(95 \% \mathrm{CI}=-11.28,-2.37$, p-value $=0.004)$ reduction in the patients' CSS from baseline to the end of study in the $\mathrm{FXCO}_{2}$ treatment group and a 4.83 (95\% CI $=-9.16,-0.51, \mathrm{p}$-value $=0.03)$ reduction in the patients' CSS in the sham-treatment group. In the patient component of the CSS, both the $\mathrm{FXCO}_{2}$ treatment and sham-treatment groups had a statistically significant reduction of the CSS. However, the between group difference of the patient CSS was -1.99 (95\% CI $=-8.15$, 4.17) which was not statistically significant with a p-value of 0.52 .

In the clinicians' twelve point CSS, there was a 0.82 increase (worsening of objective findings) in the $\mathrm{FXCO}_{2}$ treatment group $(95 \% \mathrm{CI}=-0.46,2.11, \mathrm{p}$-value $=0.20)$ and a 0.28 reduction in the sham-treatment group $(95 \% \mathrm{CI}=-1.53,0.97, \mathrm{p}$-value $=0.65)$. Neither treatment group had a statistically significant change in the clinicians' CSS from baseline to the end of the study. Additionally, the difference in the clinicians' CSS between the two treatment arms was $1.10(95 \% \mathrm{CI}=-2.87,0.68)$, which was not statistically significant with a p-value of 0.22 (Table 2). Other than complaints of transient, mild discomfort, no adverse events were reported.

\section{Discussion}

\section{Main findings}

This study showed that there was no meaningful improvement in the histopathological changes of VLS with $\mathrm{FXCO}_{2}$ laser therapy compared to sham treatment, indicating that $\mathrm{FXCO}_{2}$ is not an effective treatment for VLS. An additional significant finding is that women in both the active treatment arm and sham treatment arm experienced a statistically significant improvement in symptoms of VLS, illustrating a large placebo effect and highlighting the need for randomized, controlled trials to obtain meaningful data for the treatment of VLS.

\section{Interpretation}

In many studies, $\mathrm{FXCO}_{2}$ has been shown to be an effective treatment for genitourinary syndrome of menopause (GSM, formerly known as vulvovaginal atrophy). Specifically, randomized, controlled studies of $\mathrm{FXCO}_{2}$ have shown to significantly improve the symptoms of GSM, such as vaginal dryness and dyspareunia. ${ }^{2}$ Additionally, $\mathrm{FXCO}_{2}$ has been shown to improve the histopathologic changes of GSM, including increasing submucosal vascularity, collagen deposits and elastin fibers. ${ }^{2}$ However, in the vast majority of cases there is only mild inflammatory changes associated with GSM. In contrast, the inflammatory process in VLS, which is most likely of autoimmune etiology, is incredibly robust. The intense inflammation process in 
VLS can cause severe induration, lichenification, fissures, erosions, severe scarring, and malignant transformation. Our study demonstrated that $\mathrm{FXCO}_{2}$ does not have the ability to reverse the intense inflammation of VLS.

The results of our study contrast with several studies that have shown $\mathrm{FXCO}_{2}$ to be beneficial for women with VLS. However, there are significant methodological flaws in previous studies that must be noted. Pagano et al. performed a prospective, non-randomized, non-controlled, non-blinded study of 40 women with VLS treated with $\mathrm{FXCO}_{2} .{ }^{1}$ Thirty-seven of the 40 women also were treated for GSM with the laser. They reported a statistically significant improvement in the patients' symptoms of pruritis, dryness, and dyspareunia but it is unclear if the improvement in symptoms was related to treatment of VLS or GSM, or both. Additionally, it is possible that this improvement was due entirely to placebo effect, as our study also showed that women who received the sham treatment had a statistically significant improvement in subjective symptoms as measured by the CSS. Another study by Balchander and Nyirjesy was a retrospective study of 40 women with VLS treated with $\mathrm{FXCO}_{2}$. As in the Pagano study, the majority of participants were also treated for GSM, in this study with both $\mathrm{FXCO}_{2}$ and topical estradiol. Additionally, 38 of the 40 remained on corticosteroids throughout the treatment period. ${ }^{16}$ It is unclear if the statistically significant improvement in vaginal pain, itching, dyspareunia, and dysuria that they found was related to treatment of the VLS, GSM, or both, or was due to placebo effect, or the effects of the corticosteroid and/or estradiol. Lastly, Baggish published a retrospective case series of 27 women with biopsy proven LS treated with 3-4 treatments of $\mathrm{FXCO}_{2}$ with power settings similar to our study. He describes "visible improvement of the vulvar skin was seen in 26/27 patients, while $24 / 27$ women have had no further itching or any sort of vulvar discomfort or pain." ${ }^{1}$ Unfortunately, there was no independent corroboration of these observations nor any objective data such as post-treatment histopathology or an evaluation of post-treatment photos by an independent clinician. It should be noted that in all of the studies described above, the patients paid for their treatments- typically \$USD 500 or more per treatment and the providers (who were authors on these studies) received payment for these treatments. such, there is a concern for confirmation bias in the form of elevation of commitment bias for the investigators and cognitive dissonance bias of the subjects in all of these studies.

Additionally, there have been a few studies that have used a non-ablative neodymium: yttrium aluminum garnet (Nd:YAG) laser for VLS. Bizjak-Ogrinc and colleagues conducted a randomized, non-blinded study that compared 20 women who used corticosteroids and had three fractional Nd:YAG laser treatments to 20 women who used corticosteroids alone. Pre and posttreatment biopsies were obtained and showed no statistical difference in inflammation between groups. ${ }^{1}$

\section{Strengths and limitations}

In contrast to the aforementioned studies that have examined $\mathrm{FXCO}_{2}$ for VLS, our study is prospective, randomized, double-blind, and sham controlled. To ensure blinding, the sham treatment we chose was designed to deliver a very small amount of laser energy to this tissue to produce visible spots on the skin and creates smoke and odor from skin vaporization, but not strong enough to affect the pathological process. Blinding is especially important because, as our results demonstrated, there is a very strong placebo effect in this type of study, evidenced by a statically significant increase in the patients' subjective symptom improvement in the sham treatment arm.

Another strength of our study is use of underlying histopathologic changes as the primary endpoint. The underlying inflammation in VLS causes significant morbidity beyond the subjective symptoms of VLS, including both vulval scarring and a significant risk of malignant transformation. As such, any treatment that only improves symptoms, but does not reverse underlying histopathologic changes is inadequate. It is important to emphasize that ultrapotent corticosteroids, the current gold standard treatment for VLS, do reverse the histopathologic changes of VLS, thereby reducing additional scarring and decreasing the risk of malignant transformation. Since ultrapotent corticosteroids are readily available, any new treatment for VLS must show reduction in underlying histopathologic changes of VLS. As such, the United States FDA has recently indicated that improvement in histopathologic changes must be the primary endpoint in any investigational new drug application (IND) for VLS. The use of the CSS, a validated tool designed specifically for VLS, as 
a secondary endpoint is also a strength of the study. As it measures both patient's subjective symptoms and objective clinician finding, if it is used in a blinded study, meaningful data regarding improvement or progression of VLS can be obtained.

Another strength of our study was the elimination of confounding variables. Specifically, there was a treatment washout period and active VLS was confirmed via histopathology at the onset of the study in all study participants. Additionally, no $\mathrm{FXCO}_{2}$ treatments were administered for the treatment of GSM during the study. The specific energy settings used in the study were optimized for the treatment for VLS. Additionally, the number of treatments in this study (five) was standardized and of a significant quantity to ensure that efficacy could be demonstrated.

One weakness of this study was its relatively small sample size. However, an a priori sample size calculation determine that the selected sample was large enough to demonstrate a clinically meaningful difference in the primary endpoint (histopathologic changes of VLS). An additional limitation of this study is that rater bias was potentially introduced as several different (blinded) clinicians participated in scoring the provider section of the CSS.

\section{Conclusions}

Despite previously optimistic results in well-designed clinical trials of $\mathrm{FXCO}_{2}$ for GSM, and in non-controlled case-series for VLS, our study failed to show any significant benefit of monotherapy of $\mathrm{FXCO}_{2}$ for VLS. There may be a role for $\mathrm{FXCO}_{2}$ as an adjuvant therapy along with topical ultrapotent corticosteroids in VLS. To adequately study this, however, will require additional well-designed studies that include a sham-placebo arm, as our study demonstrated a large placebo effect.

Acknowledgements: The authors would like to thank Dr. Sarah Bedell, Dr. Sarah Cigna, Dr. Mollie Reiff, and Ms.Vaishnavi Govind for their help with data acquisition.

\section{Disclosure of Interests:}

Leia Mitchell reports no conflicts of interest.

Andrew Goldstein: Dr. Goldstein is President of the Gynecologic Cancer Research Foundation, a 501 c3 non-profit corporation, which provided partial funding for this study. He is a part-time employee of Dare Bioscience. He has received research funding from Dare Science, SST, Endoceutics, The Cellular Medicine Association, and Ipsen. He is a consultant for Ipsen, SST, and AMAG

Debra Heller reports no conflicts of interest.

Theodora Mautz reports no conflicts of interest.

So Yeon Joyce Kong is an employee of Laerdal Medical, Stavanger, Norway

Maria Sophocles is on the speakers' bureau for Duchenay, DEKA, and Cynosure

Chelsea Thorne reports no conflicts of interest.

Hillary Tolson reports no conflicts of interest.

Jill Krapf reports no conflicts of interest.

\section{Contribution to Authorship}

L.M. was responsible for data collection, interpretation of data, and manuscript preparation.

A.G. was responsible for study design, data collection, interpretation of data, and manuscript preparation and editing

C.T. was responsible for data collection and manuscript editing

J.K. was responsible for interpretation of data and manuscript preparation. 
D.H. was responsible for data collection and interpretation of data.

M.S. was responsible for data collection

H.T. was responsible for data collection and manuscript editing.

T.M. was responsible for data collection and manuscript editing.

J.K. was responsible for data collection, interpretation of data, and manuscript editing

Clinical trial registration: ClinicalTrials.gov Identifier: NCT03665584

IRB Approval: Advarra IRB: Pro00024516, March 1, 2018 (attached)

\section{Funding Sources:}

Partial funding for this study was obtained from the Gynecologic Cancer Research Foundation, a Maryland, USA 501(C) 3 non-profit corporation. Additional funding for this study was supplied by El.En Group, Florence, Italy, the manufacturer of the laser used in this study. In addition, El.En Group supplied the laser used in the study. El.En Group had no role in the analysis and interpretation of data; in the writing of the report; or in the decision to submit the article for publication.

\section{References}

1. Goldstein AT, Marinoff SC, Christopher K, Srodon M. Prevalence of vulvar lichen sclerosus in a general gynecology practice. J Reprod Med. 2005;50:477-80.

2. Cooper SM, Gao XH, Powell JJ, Wojnarowska F. Does treatment of vulvar lichen sclerosus influence its prognosis? Arch Dermatol. 2004;140:702-6.

3. Tran DA, Xiaohui T, Macri CJ, Goldstein AT, Fu SW. Lichen sclerosus: An autoimmunopathologenic aned genomic enigma with emerging genetic and immune targets. Int J Biol Sci. 2019; 15(7):1429-39.

4. Lewis FM, Tatnall FM, Velangi SS, Bunker CB, Kumar A, Brackenbury F, et al. British Association of Dermatologists guidelines for the management of lichen sclerosus 2018. British Journal of Dermatology $2018 ; 823-824$.

5. Kirtschig G, Becker K, Günthert A, Jasaitiene D, Cooper S, Chi CC, et al. Evidence-based (S3) Guideline on (anogenital) Lichen sclerosus. Journal of the European Academy of Dermatology and Venereology. 2015 Oct;29(10):e1-43.

6. Lee A, Bradford J, Fischer G. Long-term management of adult vulvar lichen sclerosus: A prospective cohort study of 507 women. JAMA Dermatol. 2015; 151(10):1061-7.

7. Furue M, Terao H, Rikihisa W, Urabe K, Kinukawa N, Nose Y, et al. Clinical dose and adverse effects of topical steroids in daily management of atopic dermatitis. The British Journal of Dermatology 2003;148:12833.

8. Hantash BM, Bedi VP, Struck SK, Chan KF. Immunohistochemical evaluation of the heat shock response to nonablative fractional resurfacing. JBO 2010;15(6):068002.

Ferrara F, Messori S, Abbenante D, Patrizi A, Bardazzi F. Fractional CO2 laser therapy of lichen sclerosus in males: a new therapeutic opportunity? Journal of Dermatological Treatment 2020;0(0):1-5.

- Gomez-Frieiro M, Laynez-Herrero E. Use of Er:YAG laser in the treatment of vulvar lichen sclerosus. International Journal of Women's Dermatology 2019;5(5):340-4.

1. Hobson JG, Ibrahim SF, Mercurio MG. Recalcitrant Vulvar Lichen Sclerosus Treated With Erbium YAG Laser. JAMA Dermatol 2019;155(2):254-6.

1. Lee A, Lim A, Fischer G. Fractional carbon dioxide laser in recalcitrant vulval lichen sclerosus. Australas J Dermatol 2016;57(1):39-43. 
1. Mendieta-Eckert M, Ocerin-Guerra I, Landa-Gundin N. Lichen sclerosus et atrophicus in a surgical scar treated with fractional laser. J Cosmet Laser Ther 2017;19(2):106-8.

1. Mendieta-Eckert M, Torrontegui Bilbao J, Zabalza Estevez I, Landa Gundin N. Treatment of Vulvar Lichen Sclerosus et Atrophicus With Fractional Carbon Dioxide Laser Therapy: A Report of 4 Cases [published online ahead of print, $2020 \mathrm{Jul}$ 14]. Tratamiento de liquen escleroso y atrofico vulvar con laser de dioxido de carbono fraccionado. Presentacion de 4 casos [published online ahead of print, 2020 Jul 14]. Actas Dermosifiliogr. 2020;S0001-7310(20)30230-1. doi:10.1016/j.ad.2019.03.021

1. Pagano T, Conforti A, Buonfantino C, Schettini F, Vallone R, Gallo A, et al. Effect of rescue fractional microablative CO2 laser on symptoms and sexual dysfunction in women affected by vulvar lichen sclerosus resistant to long-term use of topic corticosteroid: a prospective longitudinal study. Menopause 2020;27(4):418-22.

1. Balchander D, Nyirjesy P. Fractionated CO2 Laser as Therapy in Recalcitrant Lichen Sclerosus. Journal of Lower Genital Tract Disease 2020;24(2):225-228.

1. Baggish MS. Fractional CO2 Laser Treatment for Vaginal Atrophy and Vulvar Lichen Sclerosus. Journal of Gynecologic Surgery 2016;32(6):309-17.

1. Bizjak Ogrinc U, Senčar S, Luzar B, Lukanović A. Efficacy of Non-ablative Laser Therapy for Lichen Sclerosus: A Randomized Controlled Trial. J Obstet Gynaecol Can 2019;41(12):1717-25.

1. Günthert AR, Duclos K, Jahns BG, Krause E, Amann E, Limacher A, et al. Clinical scoring system for vulvar lichen sclerosus.J Sex Med. 2012 Sep;9(9):2342-50. doi: 10.1111/j.1743-6109.2012.02814.x. Epub 2012 Jul 3.

- Ruanphoo P, Bunyavejchevin S. Treatment for vaginal atrophy using microablative fractional CO2 laser: a randomized double-blinded sham-controlled trial. Menopause . 2020;27(8):858-863. doi:10.1097/GME.0000000000001542

2. Cruz VL, Steiner ML, Pompei LM, Strufaldi R, Fonseca FL, Santiago LH, et al. Randomized, doubleblind, placebo-controlled clinical trial for evaluating the efficacy of fractional CO2 laser compared with topical estriol in the treatment of vaginal atrophy in postmenopausal women. Menopause . 2018;25(1):21-28. doi:10.1097/GME.0000000000000955

2. Samuels JB, Garcia MA. Treatment to external labia and vaginal canal with CO2 laser for symptoms of vulvovaginal atrophy in postmenopausal women. Aesthet Surg J 2019; 39: 83-93.

\section{Hosted file}

BJOB Figure 1.pdf available at https://authorea.com/users/364500/articles/484892-fractionalco2-laser-for-the-treatment-of-vulvar-lichen-sclerosus-a-double-blind-sham-controlledrandomized-trial

\section{Hosted file}

BJOB Table 1.pdf available at https://authorea.com/users/364500/articles/484892-fractionalco2-laser-for-the-treatment-of-vulvar-lichen-sclerosus-a-double-blind-sham-controlledrandomized-trial 


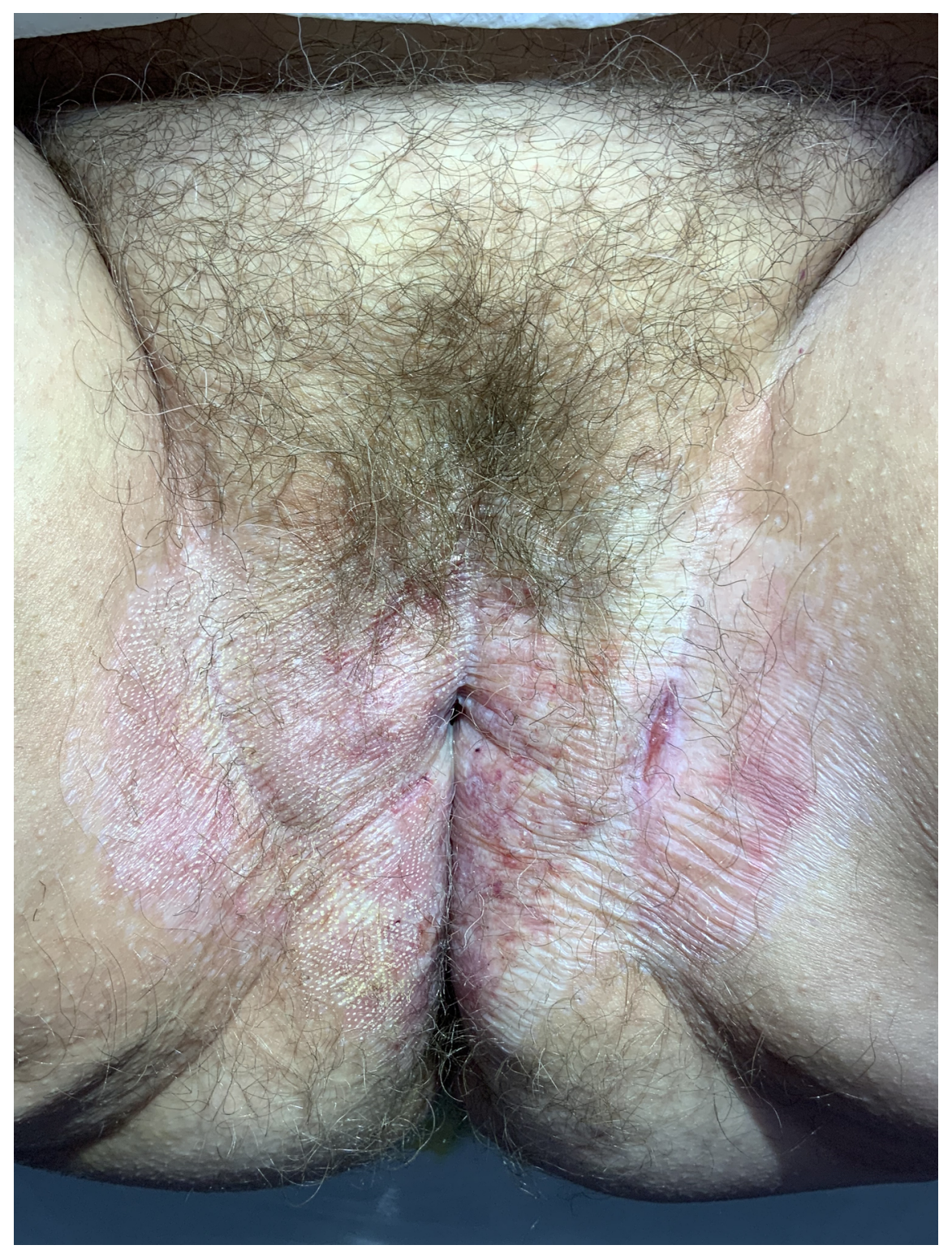


Table 2. Changes in Patient CSS, Provider CSS, and HS by Treatment

\begin{tabular}{|c|c|c|c|c|c|c|}
\hline & \multirow{2}{*}{\multicolumn{2}{|c|}{ Active treatment }} & \multirow{2}{*}{\multicolumn{2}{|c|}{ Sham treatment }} & \multirow{3}{*}{$\begin{array}{c}\text { Between Group } \\
\text { Difference } \\
(95 \% \mathrm{CI}) \\
\end{array}$} & \multirow{3}{*}{$\begin{array}{c}\mathrm{p}- \\
\text { value }\end{array}$} \\
\hline & & & & & & \\
\hline & $\begin{array}{l}\text { Crude Mean } \\
\text { (SE) }\end{array}$ & $\begin{array}{l}\text { Model-based } \\
\text { Mean } \\
(95 \% \mathrm{CI}) \\
(\mathrm{N}=17)\end{array}$ & $\begin{array}{l}\text { Crude Mean } \\
\text { (SE) }\end{array}$ & $\begin{array}{l}\text { Model-based' } \\
\text { Mean } \\
(95 \% \mathrm{CI}) \\
(\mathrm{N}=18)\end{array}$ & & \\
\hline \multicolumn{7}{|l|}{ Patients' CSS } \\
\hline Baseline Visit & $24.29(1.77)$ & $\begin{array}{l}29.45(23.84 \\
35.06)\end{array}$ & $25.14(1.72)$ & $\begin{array}{l}30.95(24.65, \\
37.24)\end{array}$ & $-0.84(-5.88,4.19)$ & 0.73 \\
\hline EOS Visit & $17.47(2.67)$ & $\begin{array}{l}22.63(15.81 \\
29.45)\end{array}$ & $20.31(2.59)$ & $\begin{array}{c}26.11(18.78, \\
33.44)\end{array}$ & $\begin{array}{c}-2.84(-10.41, \\
4.74)\end{array}$ & 0.45 \\
\hline $\begin{array}{l}\Delta \text { Baseline to } \\
\text { EOS } \\
\text { Provider CSS }\end{array}$ & $-6.82(2.17)$ & $\begin{array}{c}-6.82(-11.28,- \\
2.37)\end{array}$ & $-4.83(2.11)$ & $\begin{array}{c}-4.83(-9.16,- \\
0.51)\end{array}$ & $-1.99(-8.15,4.17)$ & 0.52 \\
\hline Baseline Visit & $7.53(0.46)$ & $6.91(5.43,8.39)$ & $9.06(0.44)$ & $8.20(6.53,9.88)$ & $\begin{array}{c}-1.53(-2.82,- \\
0.23)\end{array}$ & 0.02 \\
\hline EOS Visit & $8.35(0.58)$ & $7.73(5.99,9.47)$ & $8.78(0.56)$ & $7.92(6.03,9.82)$ & $-0.43(-2.07,1.22)$ & 0.60 \\
\hline $\begin{array}{l}\Delta \text { Baseline to } \\
\text { EOS }\end{array}$ & $+0.82(0.63)$ & $\begin{array}{c}+0.82(-0.46 \\
2.11)\end{array}$ & $-0.28(0.61)$ & $\begin{array}{c}-0.28(-1.53 \\
0.97)\end{array}$ & $+1.10(-2.87,0.68)$ & 0.22 \\
\hline \multicolumn{7}{|l|}{$\begin{array}{l}\text { Histopathology } \\
\text { scale }\end{array}$} \\
\hline Pre-treatment & $4.41(0.31)$ & $4.26(3.29,5.23)$ & $4.28(0.30)$ & $4.07(2.98,5.16)$ & $+0.13(-0.74,1.01)$ & 0.76 \\
\hline Post-treatment & $4.29(0.38)$ & $4.14(3.09,5.19)$ & $4.33(0.37)$ & $4.13(2.98,5.28)$ & $-0.04(-1.12,1.04)$ & 0.94 \\
\hline$\Delta$ Pre to Post & $-0.12(0.44)$ & $\begin{array}{c}-0.12(-1.01, \\
0.78)\end{array}$ & $0.06(0.42)$ & $\begin{array}{c}+0.06(-0.81, \\
0.92)\end{array}$ & $-0.17(-1.41,1.06)$ & 0.78 \\
\hline
\end{tabular}

